\title{
Magnetic field contributes to the cellular uptake for effective therapy with magnetofection using plasmid DNA encoding against Mcam in B16F10 melanoma in vivo
}

\begin{abstract}
Aim: We explored the distribution and cellular uptake of intratumorally injected SPIONs-PAA-PEI-pDNA (magnetofection complexes), and antitumor effectiveness of magnetofection with plasmid DNA encoding short hairpin RNA (shRNA) against Mcam (pDNA ${ }^{\text {anti-MCAM}) . ~ M a t e r i a l s ~ \& ~ m e t h o d s: ~ A n a l y s e s ~ w e r e ~ m a d e ~ b a s e d ~ o n ~ t h e ~ h i s t o l o g y, ~}$ ultrastructure and quantitative measurements of magnetofection complexes, and quantification of the antitumor effectiveness in B16F10 melanoma in vivo. Results: Injected magnetofection complexes were distributed around the injection site. Exposure of tumors to external magnetic field contributed to the uptake of magnetofection complexes from extracellular matrix into melanoma cells. Three consecutive magnetofections of tumors with pDNA anti-MCAM resulted in significant reduction of tumor volume. Conclusion: Magnetofection is effective for gene delivery to melanoma tumors, but requires a magnetic field for cellular uptake and antitumor effect.
\end{abstract}

First draft submitted: 27 September 2015; Accepted for publication: 5 January 2016; Published online: 8 March 2016

Keywords: cellular uptake $\bullet$ intratumoral injection $\bullet$ magnetofection $\bullet$ MCAM $\bullet$ murine melanoma tumor $\bullet$ SPIONs • therapeutic plasmid DNA

Magnetofection is a promising nonviral gene delivery method, which could in the future be used as a complementary method to the conventional cancer treatments, due to its effectiveness, noninvasiveness and painless procedure [1]. In the recent decade a significant progress in the field of magnetic nanoparticle formulations, their biocompatibility and applications of magnetofection in vitro and in vivo was made [2]. Recently, we developed unique superparamagnetic iron oxide nanoparticles (SPIONs) coated with polyacrylic acid (PAA) and functionalized with polyethylenimine (PEI) for further binding of plasmid DNA ( $\mathrm{pDNA}$ ). In the process of developing SPIONs-PAA-PEI complexes we tested different polymers for SPIONs coating besides PAA, namely aminopropyl triethoxy silane (APS), (aminoethylamino)propyl triethoxy silane (APMS) and citric acid
(CA). Among all tested coatings only PAA proved to be effective in transfection of cells in vitro [Unpublished Data]. Our in vitro and in vivo studies demonstrated SPIONs-PAAPEI complexes as safe and efficient carriers of reporter and therapeutic plasmids, encoding either IL-12 ( $\mathrm{pDNA}^{\mathrm{IL}-12}$ ) or shRNA against Mcam (pDNA ${ }^{\text {anti-MCAM }}$ ) [1,3-4]. As one of the few research groups we demonstrated antitumor effect in murine tumor models in vivo after magnetofection with therapeutic plasmids [1,3]. Namely, three consecutive treatments with $\mathrm{pDNA}^{\mathrm{IL}-12}$ or $\mathrm{pDNA}^{\text {anti-MCAM }}$ lead to a significant growth delay of mammary adenocarcinoma and highly metastatic melanoma, respectively $[1,3]$. In these two studies we even proved that this treatment is safe, since no systemic toxicity during the treatment was noticed, and also intraperitoneal injection of low doses of SPIONs-PAA-PEI-
Lara Prosen', Samo Hudoklin², Maja Cemazar ${ }^{1,3}$, Monika Stimac', Ursa Lampreht Tratar', Maja Ota ${ }^{4}$, Janez Scancar ${ }^{5}$, Rok Romih ${ }^{2}$ \& Gregor Sersa ${ }^{*, 1}$

'Department of Experimental Oncology, Institute of Oncology Ljubljana,

Zaloska 2, SI-1000 Ljubljana, Slovenia ${ }^{2}$ Institute of Cell Biology, Faculty of Medicine, University of Ljubljana, Vrazov $\operatorname{trg}$ 2, SI-1000 Ljubljana, Slovenia ${ }^{3}$ Faculty of Health Sciences, University of Primorska, Polje 42, SI-6310 Izola, Slovenia

${ }^{4}$ Department of Pathology, Institute of Oncology Ljubljana, Zaloska 2, SI-1000 Ljubljana, Slovenia

${ }^{5}$ Department of Environmental Sciences, Jozef Stefan Institute, Jamova 39, SI-1000 Ljubljana, Slovenia *Author for correspondence: Tel. \& Fax: +38 615879434 gsersa@onko-i.si 
pDNA (1.2-2-2 mg/kg) did not cause any differences in the concentration of iron within the organs $[1,3]$.

In order to explore, gain and update the knowledge about the functioning of magnetic nanoparticles on the nano- and micrometric scale, further studies are needed. To date, extensive in vitro studies on the uptake, trafficking and accumulation of magnetic nanoparticles in the cells after magnetofection were already done $[2,5]$. The research groups demonstrated that the uptake mechanism of magnetic nanoparticles and its efficiency are size, shape, coating and concentration dependent, and in addition also cell type dependent [3,6-7]. Specifically, the uptake of bare and surface modified SPIONs was carried out by clathrin- or caveolin mediated endocytosis and phagocytosis, respectively [3,8-11]. Without suitable surface modification SPIONs remain within maturing endosomes until digested to the elementary iron ions as a consequence of acidic and reducing conditions in lysosomes $[12,13]$. However, the surface modification of SPIONs with endosomolytic polymers, such as PAA and PEI, enables endosomal escape of nucleic acids by the proton sponge effect [3,14-18].

In contrast to in vitro studies, the studies on the uptake, trafficking and accumulation of magnetic nanoparticles in tumors in animal models in vivo after magnetofection are scarce and, therefore, required. With the outcomes of these studies, we could get more insight into the mechanisms of action, and possible improvements of the methods, and in the end, to bring magnetofection closer to the clinical applications. To date, only two research groups studied the mechanism of the internalization of intratumorally (it.) delivered coated magnetic nanoparticles into murine mammary adenocarcinoma and hypopharyngeal carcinoma in mice in vivo, and demonstrated the accumulation of coated magnetic nanoparticles into large clusters within cytoplasmic vesicles [19-21].

Therefore, the aim of this study was to explore the distribution, accumulation, uptake and the consequent therapeutic effect of it. injected SPIONs-PAAPEI-pDNA anti-MCAM (magnetofection complexes) in the presence (magnetofection) or absence of an external magnetic field (nanofection) in murine melanoma tumor model in vivo. The therapeutic plasmid DNA $\mathrm{pDNA}^{\text {anti-MCAM }}$ was selected, since MCAM has been previously described as a potential target in anticancer therapies in mice [1,22-24]. The distribution and cellular uptake of magnetofection complexes were examined by transmission electron microscopy (TEM), the accumulation of magnetofection complexes in murine melanoma was quantitatively determined by inductively coupled plasma mass spectrophotometer (ICP-MS), and the potential antitumor effect of triple consecutive magnetofections with $\mathrm{pDNA}^{\text {anti-MCAM }}$ was determined by monitoring tumor growth and analyzing immunohistochemically stained tumor sections. To summarize, the focus of this study was more on the functionality of prepared magnetofection complexes rather than their physicochemical properties.

The findings of this study indicate that magnetofection is effective for gene delivery to melanoma tumors in vivo, but requires a magnetic field for the cellular uptake and antitumor effect.

\section{Materials \& methods}

SPIONs synthesis \& preparation of magnetofection complexes with bound therapeutic plasmid DNA

SPIONs were synthesized by alkaline co-precipitation of ferrous and ferric sulfates $\left(\mathrm{FeSO}_{4} \cdot 7 \mathrm{H}_{2} \mathrm{O}, 98 \%\right.$ and $\left[\mathrm{Fe}_{2}\left(\mathrm{SO}_{4}\right)_{3} \cdot \mathrm{xH}_{2} \mathrm{O}\right]$ ) (Alfa Aesar, Ward Hill, MA) in an aqueous solution according to the Massart method as described in our previous article [3]. Magnetofection complexes were prepared as follows [3]: immediately after SPIONs synthesis the SPIONs were coated with $45 \%(\mathrm{w} / \mathrm{w})$ water solution of $\mathrm{pH}$-responsive anionic polymer poly(acrylic acid, sodium salt) (PAA) with molecular weight of $8 \mathrm{kDa}$ (Sigma Aldrich, MO, USA) to obtain SPIONs-PAA. SPIONs-PAA were functionalized directly prior to the experiments with branched cationic polymer polyethylenimine (PEI) with molecular weight of $25 \mathrm{kDa}$ (Sigma Aldrich) in the mass ratio 0.6:1 to obtain SPIONs-PAA-PEI. Therapeutic plasmid DNA (pDNA) was used for binding to SPIONs-PAA-PEI to obtain SPIONs-PAA-PEI-pDNA in the mass ratio $0.6: 1: 1[3,4]$. This mass ratio was proved to be the most suitable in our previous studies, according to the results of cytotoxicity and transfection efficiency assays [3,4]. Moreover, from all tested mixing sequences the most effective transfection of cells in vitro was obtained by mixing firstly SPIONs-PAA and PEI, and subsequently adding pDNA into the mixture [Unpublished Data]. See the experimental workflow (Figure 1A).

According to above described preparation procedure, the synthesized SPIONs have a hydrodynamic size of $39.6 \pm 1.9 \mathrm{~nm}$, polydispersity index less than 0.1 (indicating on narrow particle size distribution and monodispersity) and zeta potential of $-24 \pm 2 \mathrm{mV}$ at $\mathrm{pH} 9.5$. SPIONs coated with PAA have a hydrodynamic size of $56.5 \pm 1.7 \mathrm{~nm}$, polydispersity index less than 0.1 and zeta potential of $-47 \pm 2 \mathrm{mV}$ at $\mathrm{pH} 8.5$ (indicating on the stabilization of magnetic fluid). SPIONs-PAA-PEI have zeta potential of $20 \pm 1 \mathrm{mV}$ at $\mathrm{pH} 8.0$, indicating on the positive surface charge, which is crucial for the further binding of pDNA. Finally, SPIONs-PAA-PEIpDNA measure approx. 200-400 $\mathrm{nm}$ in diameter and have the zeta potential of $8 \pm 1 \mathrm{mV}$ at $\mathrm{pH}$ 8.0. 


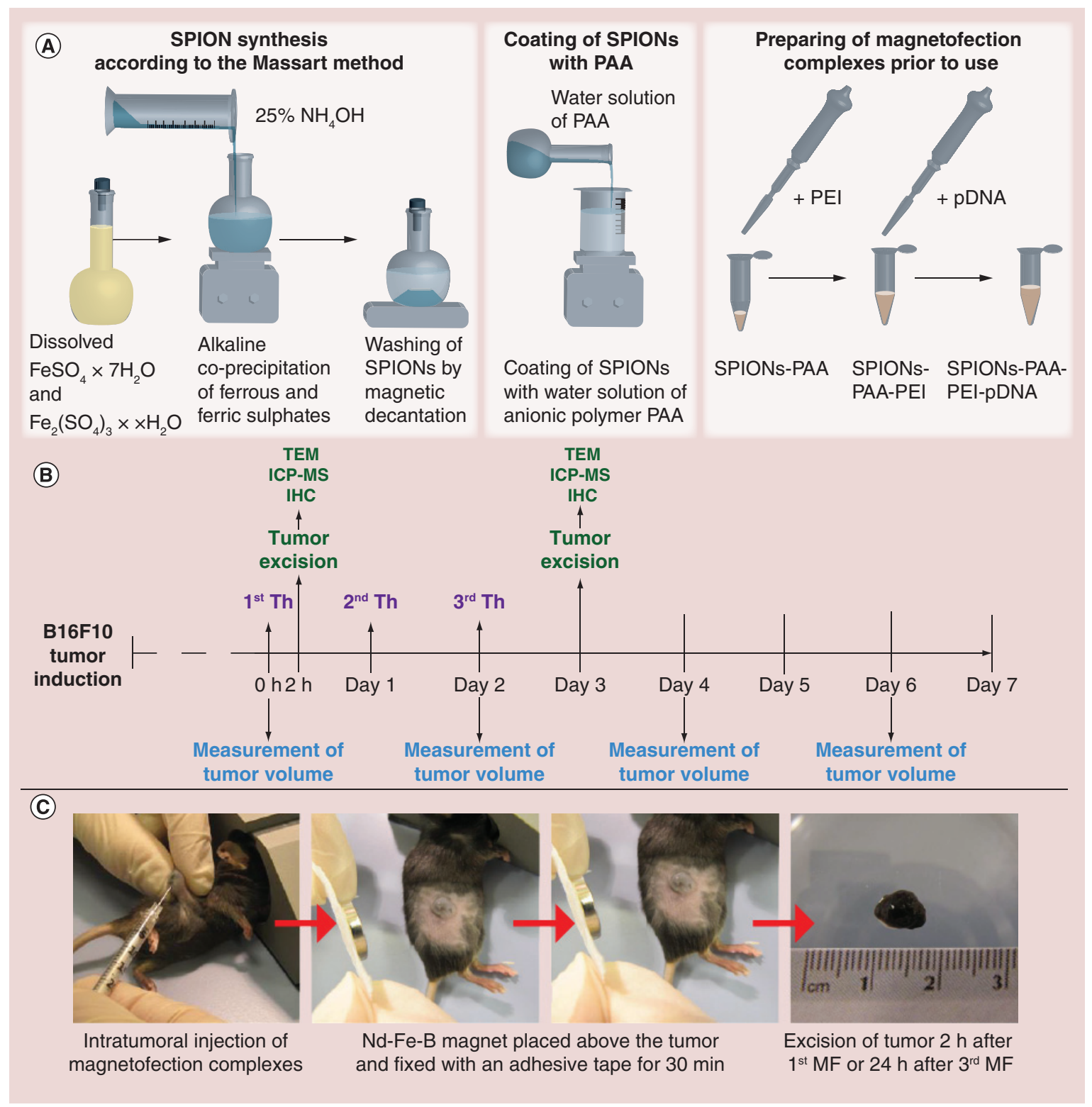

Figure 1. The experimental workflow. (A) The scheme of SPION synthesis and immediate coating with PAA, and further preparation of magnetofection complexes prior to use. (B) B16F10 tumors were subcutaneously induced by injecting a suspension of B16F10 cells into the shaved right flank of a mouse. When tumors reached $40 \mathrm{~mm}^{3}$, mice were randomly divided into four experimental groups and subjected to the therapy (Th) for 3 consecutive days. The mice from each experimental group were sacrificed $2 \mathrm{~h}$ after the $1 \mathrm{st}$ Th and $24 \mathrm{~h}$ after the $3 \mathrm{rd}$ Th. Their tumors were excised and prepared for further examination by TEM, ICP-MS and ICH. The tumor growth was monitored in six mice per experimental group, until the tumors in the CTRL reached $350 \mathrm{~mm}^{3}$, and then the animals were euthanized. (C) The demonstration of MF of B16F10 tumor in mouse in vivo and the appearance of excised tumor.

CTRL: Control group; ICH: Immunohistochemical staining; ICP-MS: Inductively coupled plasma mass spectrophotometer; MF: Magnetofection; PAA: Polyacrylic acid; SPION: Superparamagnetic iron oxide nanoparticle; TEM: Transmission electron microscopy.

In this study, we bound a therapeutic plasmid DNA, which encodes short hairpin RNA (shRNA) against Mcam (pDNA ${ }^{\text {anti-MCAM}}$ ) under the control of human U6 promoter to allow the RNA polymerase III-controlled expression of shRNA in mammalian cells (pENTR/ U6 anti-MCAM; constructed in our research group)

(2909 bp) [1]. The plasmid DNA was isolated using a NoEndo JETSTAR ENDOTOXIN-FREE MEGA/ GIGA Kit (Genomed, Löhne, DE) and diluted in endotoxin-free water to a concentration of $1 \mu \mathrm{g} / \mu \mathrm{l}$. The SPIONs-PAA-PEI-pDNA ${ }^{\text {anti-MCAM }}$ complexes were generally termed as magnetofection complexes. 
Animal models \& induction of tumors

Female C57Bl/6 mice (Harlan Laboratories, Udine, IT) were housed in pathogen-free conditions at temperature $20-24^{\circ} \mathrm{C}$, relative humidity $55 \pm 10 \%$ and $12 \mathrm{~h}$ light/ dark cycle. Food and water were provided ad libitum. All procedures were performed in compliance with the guidelines for animal experiments of the EU directive (2010/63/EU) and the permission from the Veterinary Administration of the Ministry of Agriculture, Forestry and Food of the Republic of Slovenia (permission no. 34401-4/2012/2).

Animals were 12 weeks old when melanoma B16F10 tumors were induced subcutaneously. For the tumor induction, a suspension of $1 \times 10^{6} \mathrm{~B} 16 \mathrm{~F} 10$ cells (American Type Culture Collection [ATCC], Manassas, VA, USA) was prepared from a cell culture in vitro in $100 \mu \mathrm{l}$ of physiological solution and injected into the shaved right flank of a mouse. When B16F10 tumors reached $40 \mathrm{~mm}^{3}$, mice were randomly divided into four experimental groups and subjected to a specific experimental protocol for 3 consecutive days. See the experimental workflow (Figure 1).

\section{Nanofection \& magnetofection of tumors}

The first (CTRL) and second (MAGNET) groups of animals were injected it. with $40 \mu \mathrm{l}$ of distilled water. The third (nanofection; NF) and fourth (magnetofection; MF) groups of animals were injected it. with $40 \mu \mathrm{l}$ of magnetofection complexes, containing $15 \mu \mathrm{g}$ of pDNA anti-MCAM $(7.81 \mathrm{pmol})$. The injections were performed slowly (lasting $\sim 5 \mathrm{~s} ; 8 \mu \mathrm{l} / \mathrm{s}$ ), using $0.3 \mathrm{ml}$ insulin syringes with 28 -gauge needles (Terumo, Tokyo, JP). Immediately after it. injection Neodymium-IronBoron permanent magnets (Nd-Fe-B magnets with surface magnetic flux density of $403 \mathrm{mT}$ and magnetic gradient of $38 \mathrm{~T} / \mathrm{m}$; Supermagnete, Uster, $\mathrm{CH}$ ) were fixed above the tumors of the second (MAGNET) and fourth (MF) group of animals with an adhesive tape (Micro-pore plaster, $25 \mathrm{~mm} \times 9.1 \mathrm{~m}$, Tosama, Vir, SI) for $30 \mathrm{~min}$. The therapies in all groups of animals were performed for 3 consecutive days. See the experimental workflow (Figure 1B \& C).

The animals were kept under inhalation anesthesia with $1.5-3 \%$ vapor set of isoflurane (Isoflurane, Torrex Chiesi Pharma GmbH, Vienna, AU) with an oxygen flow of $1 \mathrm{l} / \mathrm{min}$ during it. injection and during the exposure of the tumors to the magnet. General anesthesia was induced and maintained by placing mice in a plastic chamber.

\section{Monitoring of tumor growth}

Tumor growth was monitored in six mice per experimental group, which tumors were not excised. The tumors were measured in three orthogonal diameters with a Vernier caliper, and their volumes were calculated using the equation $\mathrm{V}=\mathrm{a} \times \mathrm{b} \times \mathrm{c} \times \pi / 6$. The tumor growth delay for each experimental group was calculated as the difference in tumor doubling times of experimental and control group. Tumor doubling time is the number of days in which the initial tumor volume $\left(40-50 \mathrm{~mm}^{3}\right)$ doubles. The control of MAGNET, NF and MF groups of animals is only water injected into the tumors without magnet exposure (CTRL). On the day of tumor measurement, animals were also weighed, and their weight was used as a general index of systemic toxicity. Tumor volume was measured every 2 days until the tumors in the control group (CTRL) reached $350 \mathrm{~mm}^{3}$, and then the animals were euthanized. See the experimental workflow (Figure 1B).

\section{Inductively coupled plasma mass spectrophotometry}

For the determination of iron in B16F10 tumors by Inductively coupled plasma mass spectrophotometry (ICP-MS), six mice per experimental group were sacrificed, the first three $2 \mathrm{~h}$ after the 1st therapy and the second three $24 \mathrm{~h}$ after the $3 \mathrm{rd}$ therapy. See the experimental workflow (Figure 1B). The excised B16F10 tumors were weighed and mechanically disaggregated into two fractions (cells and extracellular matrix) by nylon cell strainer with $40 \mu \mathrm{m}$ mesh size (Falcon Cell Strainer, NY, USA). The upper phase on strainer represented noncellular fraction (extracellular matrix) and the fraction, which passed through $40 \mu \mathrm{m}$ sized nylon mesh represented a cellular fraction containing the tumor and stromal cells. Thereafter, the concentration of iron was determined by ICP-MS in both fractions. The amount of iron for each experimental group was normalized to the amount of iron measured in the control group (CTRL). The accumulation of iron in NF and MF experimental groups after the 1st and 3rd therapy was calculated according to the iron determined by ICP-MS in one and three doses of injected magnetofection complexes, respectively.

\section{Transmission electron microscopy}

For transmission electron microscopy (TEM), two mice per experimental group were sacrificed at the same time points as for the determination of iron by ICP-MS. See the experimental workflow (Figure 1B). The excised tumors were immediately submerged into $4 \%$ paraformaldehyde and $2.5 \%$ glutaraldehyde in $0.1 \mathrm{M}$ cacodylate buffer at room temperature for 20 min. Then, tumors were cut into two halves in the fixative and each half further into four pieces with the cutting plane being perpendicular to the first cutting plane. The fixation continued at $4^{\circ} \mathrm{C}$ for the total of 
$3 \mathrm{~h} 45 \mathrm{~min}$. Postfixation was performed in 1\% osmium tetroxide in $0.1 \mathrm{M}$ cacodylate buffer for $2 \mathrm{~h}$, followed by dehydration in graded ethanol and embedding in Epon 812 resin.

Semithin $(1 \mu \mathrm{m})$ sections were cut and examined by light microscope (Carl Zeiss AG, Axiolmager. Z1, Oberkochen, DE). Ultrathin sections were made from three regions of tumor: at the injection site, in the center and at the rim of tumor. Ultrathin sections were then counterstained with uranyl acetate and lead citrate and examined under TEM (CM100, Philips, Amsterdam, NL) running at $80 \mathrm{kV}$.

\section{Histology \& immunohistochemistry}

For the histology and immunohistochemical staining two mice per experimental group were sacrificed at the same time points as for the other analyses. See the experimental workflow (Figure 1B). The excised tumors were fixed in $\mathrm{ICH}$ zinc fixative (BD Pharmingen, BD Biosciences) overnight and embedded in paraffin. Four consecutive $2-\mu \mathrm{m}$ thick sections were cut from the center of each paraffin block. The first section was stained with Perls' Prussian blue histochemical method and the second with hematoxylin and eosin (HE). The third and the fourth sections were used for immunohistochemical staining of CD31positive blood vessels and Ki-67-positive cells (a cellular marker for proliferation). The sections were incubated with rabbit polyclonal antibodies against murine CD31 (ab28364, Abcam, MA, USA) at a dilution 1:1000 or rabbit monoclonal antibodies against Ki-67 (clone SP6, Thermo Fisher Scientific, MA, USA) at dilution 1:1200. Then a peroxidase-conjugated streptavidin-biotin system (Anti-Goat HRP-DAB Cell $\&$ Tissue Staining Kit, R\&D Systems) was used as the colorogenic reagent followed by hematoxylin counterstaining.

The images of Perl's Prussian blue, HE and immunohistochemically stained tumor sections were captured with a digital camera (DP72 CCD, Olympus, Hamburg, DE) connected to a microscope (BX-51, Olympus). The Perl's Prussian blue stained tumor sections were examined for the presence and distribution of iron. From HE stained tumor sections, the percent of necrosis was estimated by three independent observers in a blind fashion. From the immunohistochemically stained tumor sections, five images of viable tumor tissue per each mouse in every experimental group and in both time points were analyzed under the light microscope. By counting CD31-positive blood vessels and Ki-67-positive cells on the acquired images, the percentage of blood vessels and proliferating cells, respectively, normalized to the control group (CTRL) were determined.

\section{Statistical analysis}

All quantitative data were statistically analyzed by SigmaPlot 12 (Systat Software, CA, USA) and presented as arithmetic mean and standard errors $(A M \pm S E M)$. One-way analysis of variance followed by the HolmSidak test was used for the multiple comparisons. Student's t-test was used to evaluate the differences among NF and MF. The significance (level alpha) was set to 0.05 . Values of $p<0.05$ were considered to be statistically significant.

\section{Results \& discussion}

In the present study, we examined nonviral gene delivery method, that is, magnetofection, for the therapy of melanoma tumors in vivo with $\mathrm{pDNA}^{\text {anti-MCAM }}$ targeting MCAM, which is a potential target in the therapy of melanoma. The experiments, examining the distribution, accumulation, uptake and the consequent therapeutic effect of it. injected SPIONs-PAAPEI-pDNA anti-MCAM (magnetofection complexes) in the presence or absence of an external magnetic field were performed on B16F10 murine melanoma, which is widely used tumor model for the evaluation of anticancer therapies [25]. We divided our study into four sections in order to cover every distinct aspect of treatment: the distribution of it. injected magnetofection complexes in subcutaneously induced B16F10 tumors; the quantity of magnetofection complexes internalized from the extracellular matrix into the cells; the type of cells in B16F10 tumor, which predominantly uptake magnetofection complexes; and finally the antitumor effectiveness of magnetofection complexes with bound therapeutic plasmid DNA.

\section{The distribution of intratumorally injected} magnetofection complexes in B16F10 tumor Therapeutic effect depends on the distribution of injected therapeutic substance in the tumor. Therefore, it is important for the injected therapeutic substance to distribute throughout the whole tumor and reach as many tumor cells as possible [26].

In order to examine the distribution of magnetofection complexes within the tumor tissue after it. injection, paraffin tumor sections were stained with Perl's Prussian blue (Figure 2A). The staining showed that 2 $\mathrm{h}$ after 1st magnetofection the it. injected magnetofection complexes were gathered and accumulated at the injection site, while $24 \mathrm{~h}$ after $3 \mathrm{rd}$ magnetofection the accumulation was reduced, but still present. This could imply that magnetofection complexes distributed over the tissue within $24 \mathrm{~h}$ after it. injection or magnetofection complexes already started to degrade under the acidic conditions, thus the sensitivity of Perl's Prussian blue stain became too low, or magnetofection com- 


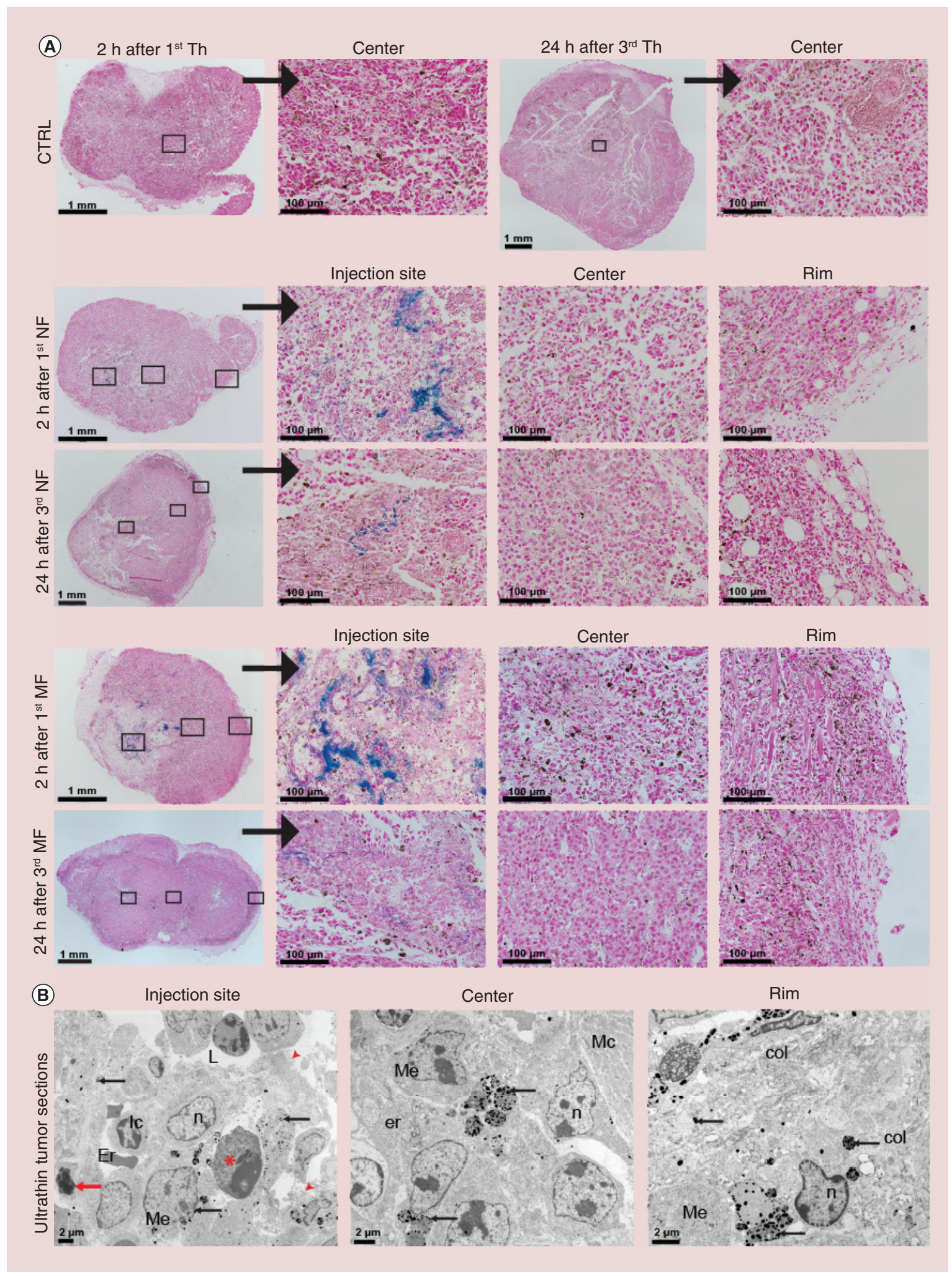

Figure 2. The distribution of intratumoral injected magnetofection complexes in B16F10 tumor. (A) The micrographs of B16F10 tumor sections stained with Perl's Prussian blue $2 \mathrm{~h}$ after $1 \mathrm{st}$ Th and $24 \mathrm{~h}$ after 3rd injection of water (CTRL), NF and MF. (B) Transmission electron microscopy micrographs of ultrathin sections cut at the injection site, in the center and at the rim of B16F10 tumor. At the injection site, the tumor tissue is composed of various types of cells, which are loosely connected and frequently undergo apoptosis or necrosis (continued on facing page). 
Figure 2 (cont.). The distribution of intratumoral injected magnetofection complexes in B16F10 tumor. At the injection site, MF complexes are seen (red arrow). In the center and at the rim of tumor, the variety of cells is still seen; however, the amount of intracellular lumen is reduced in the comparison with the sections cut at the injection site. Black arrows: melanosomes; red asterisk: apoptotic cell; red arrowheads: fragments of necrotic cells. col: Collagen; CTRL: Control group; Er: Erythrocyte; Ic: Immune cell; L: Lumen; Mc: Muscle cell; Me: Melanoma cell; MF: Magnetofection; n: Nucleus; NF: Nanofection; Th: Therapy.

plexes were washed out by tumor capillaries. In order to explore if the distribution or tissue penetration of magnetofection complexes under the influence of external magnetic field occurred, we examined also the remote sites from the injection, namely the central part of the tumor and along their rim. The staining was negative at both remote sites and both time points, thus indicating on limited distribution and tissue penetration of magnetofection complexes. This might be comprehensible, since diffusion, distribution and the uptake of macromolecules into the cells in tumors could be affected by necrosis and macrophage infiltration, increased interstitial fluid pressure and the amount of extracellular matrix components [27]. Additionally, we noticed that at the injection site the tumor tissue was less dense in the comparison with more remote sites. This could probably be due to the mechanical harms made by the needle, and the injected volume of water or suspension of magnetofection complexes.

Further, we examined ultrathin sections with TEM in order to directly visualize magnetofection complexes and their ultrastructural distribution within the tumor after magnetofection. These analyses confirmed the histological observations regarding the less dense tissue at the injection site in the comparison with more tight-appearing tissue derived from the center and the rim of tumor (Figure 2B). Moreover, TEM showed that less dense tissue at the injection site had more necrotic cells than other regions of tumor. The magnetofection complexes were noticed at the injection site.

From the results obtained in this section we can conclude that for achieving the distribution of magnetofection complexes throughout the whole tumor area after magnetofection, the it. injection of magnetofection complexes should be performed at multiple tumor application sites and with consecutive daily repetitions.

\section{The accumulation of magnetofection} complexes: cells versus extracellular matrix The effective and safe delivery of genetic material into target cells still remains a challenge in the development of nonviral gene therapy strategies. The effective uptake of magnetofection complexes in the different cell types in vitro after magnetofection was already demonstrated in our previous study by TEM and ICP-MS quantification method [3]. However, we lack the evidence on the uptake of magnetofection complexes from the extracellular matrix into the cells after magnetofection of tumors in vivo. Therefore, we examined ultrathin sections of tumors by TEM and quantitatively evaluated the accumulation of magnetofection complexes in B16F10 tumors by measuring the amount of iron. We compared quantitative data also among nanofection and magnetofection.

With TEM micrographs we proved that $2 \mathrm{~h}$ after 1 st and $24 \mathrm{~h}$ after $3 \mathrm{rd}$ magnetofection of B16F10 tumors, the it. injected magnetofection complexes were present in both, the extracellular matrix and cells (Figure 3A). The quantitative data obtained by ICP-MS quantification method demonstrated effective and comparable accumulation of magnetofection complexes in B16F10 tumors after nanofection and magnetofection (Figure 3B). Actually, 79\% (corresponding to $6.5 \pm$ $0.6 \mu \mathrm{g} \mathrm{Fe}$ ) and $87 \%$ (corresponding to $7.2 \pm 0.8 \mu \mathrm{g}$ $\mathrm{Fe}$ ) of total it. injected iron (which was present in magnetofection complexes) accumulated in the tumors after three consecutive nanofections or magnetofections, respectively. However, others quantified even $90 \%$ uptake of magnetic nanoparticles into murine mammary adenocarcinoma $4 \mathrm{~h}$ post it. injection, but in contrast to us they used TEM images for the quantification, which was not as accurate as our ICP-MS quantification method [20]. In another study a considerably lower uptake of aminosilan coated SPIONs was demonstrated, namely $65 \%$ of injected dose was present intratumorally $15 \mathrm{~min}$ after it. treatment of prostate cancer in rat [28]. However, the differences in the percentage of accumulated magnetofection complexes in tumors could be due to the different tumor models or type of magnetofection complexes used, as well as the presence or absence of an external magnetic field.

In addition, we noticed that more iron accumulated in the tumors after third than after one application, namely for $23 \%$ after both, nanofection and magnetofection (Figure 3B). We considered that this could be due to the smaller size of tumor $\left(40 \mathrm{~mm}^{3}\right)$ at the beginning of the 1st therapy. Subsequently the injected magnetofection complexes could not retain in the tumor due to the pressure, and therefore spread peritumorally. By ICP-MS only the amount of iron that retained in the tumor was determined.

Further, as we compared the accumulation efficiencies obtained after nanofection and magnetofection we noticed that the exposure of tumors to an external magnetic field post it. injection of magnetofection complexes did not increase the accumulation of iron 


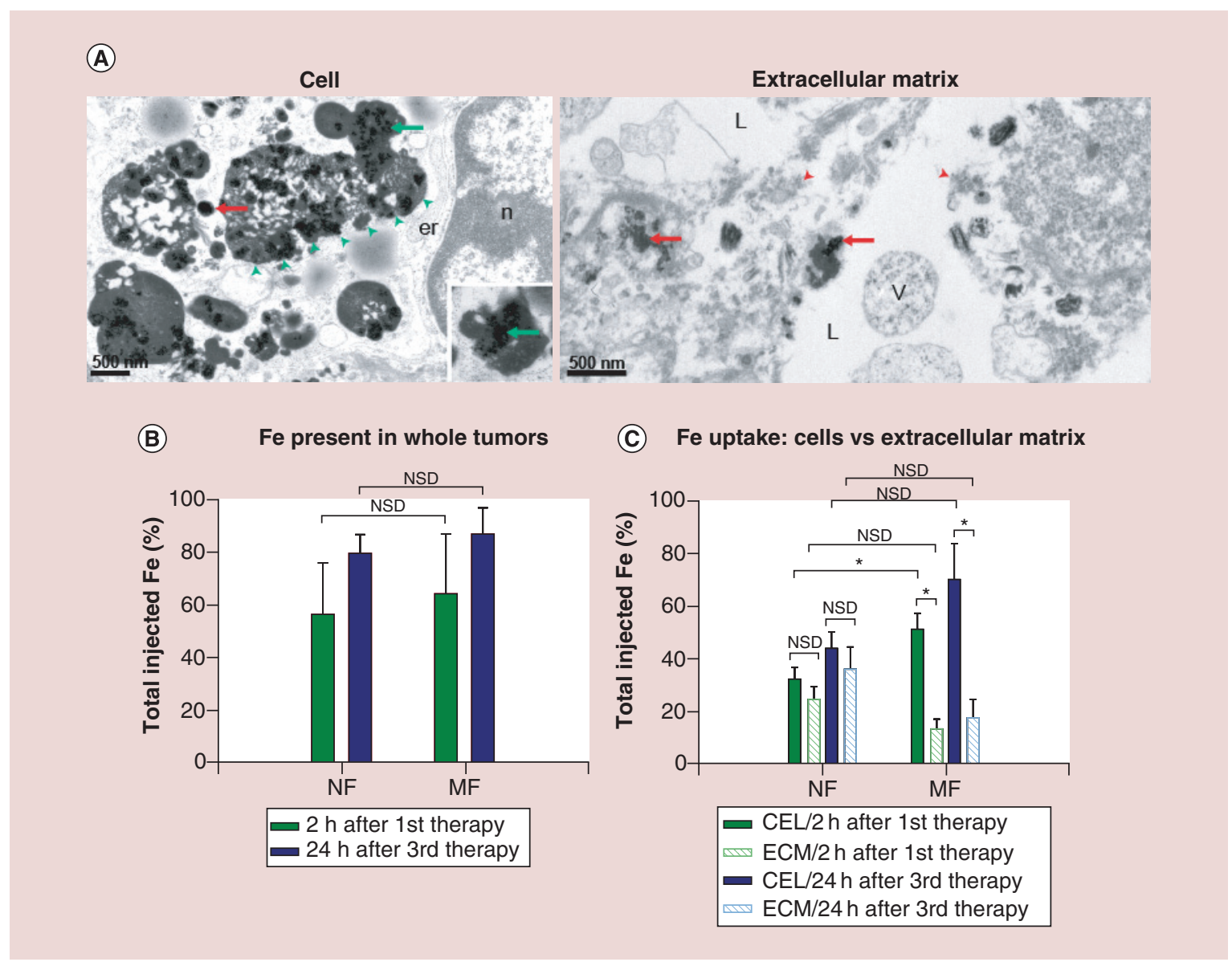

Figure 3. The accumulation of magnetofection complexes in B16F10 tumor after intratumoral injection under the absence (nanofection) or presence (magnetofection) of an external magnetic field. (A) The most representative transmission electron microscopy micrographs of magnetofection complexes present in the cell and extracellular matrix after 3rd magnetofection. In the cell, magnetofection complexes can be seen as individual (red arrow) or in aggregates (green arrowheads) with electron dense cores (green arrows). The inset presents higher magnification of the magnetofection complex in the cell. In the extracellular matrix, magnetofection complexes (red arrows) can be seen free in the lumen or as a part of cellular debris derived from cell necrosis (red arrowheads). (B) The percentage of iron ( $\mathrm{Fe}$ ), present in the whole tumors $2 \mathrm{~h}$ after $1 \mathrm{st}$ and $24 \mathrm{~h}$ after $3 \mathrm{rd}$ therapy with NF or MF. (C) The percentage of Fe uptake in CEL and ECM $2 \mathrm{~h}$ after 1st and $24 \mathrm{~h}$ after 3rd therapy with NF or MF. The asterisk indicates statistically significant differences $\left({ }^{*} \mathrm{p}<0.05\right)$. All quantitative data are expressed as arithmetic mean and standard error.

CEL: Cells; ECM: Extracellular matrix; er: Endoplasmic reticulum; Ic: Immune cell; L: Lumen; Mc: Muscle cell; Me: Melanoma cell; MF: Magnetofection; n: Nucleus; NF: Nanofection; NSD: No statistically significant differences; V: Vesicle.

in whole tumors (Figure 3B). This is not supported by previous studies, which demonstrated a significant antitumor effect of magnetic nanoparticles with bound therapeutic plasmid DNA only in the presence of external magnetic field [3,29]. Therefore, to univocally demonstrate that magnetofection complexes are present in the cells in tumors only after the exposure of tumor to the external magnetic field, we separated cells from the extracellular matrix with mechanical disaggregation of tumors. The quantification by ICP-MS method proved a pronounced difference between nanofection and magnetofection. In the tumors exposed to an external magnetic field immediately after it. injection of magnetofection complexes (magnetofection), a significant, $50 \%$ higher accumulation of iron in the cells was determined, compared with the tumors without an exposure to magnetic field (nanofection) (Figure 3C). Overall, it seems that the main point of the exposure of tumors in vivo to an external magnetic field after it. injection is not preventing the washing out of magnetofection complexes from the tumor, but contributing to the pronounced cellular uptake of magnetofection complexes from the extracellular matrix.

To the best of our knowledge, this is the first study, in which the cells were separated from the extracellular matrix after magnetofection, and the quantification of 
accumulated iron in both fractions was determined. In the time dependent study of Giustini et al. [19,20] it was shown that murine breast adenocarcinoma cells internalized the majority of magnetic nanoparticles $3 \mathrm{~h}$ post it. injection. In our study we measured approximately $30 \%$ of total injected iron intracellularly after nanofection, and $50 \%$ after magnetofection already $2 \mathrm{~h}$ post it. injection of magnetofection complexes (Figure 3B). It is important to highlight that in the contrary to the other studies [19,21], we used quantitative ICP-MS method for the precise determination of injected iron.

The obtained data clearly demonstrate that the exposure of tumors to an external magnetic field for 30 min immediately after it. injection of magnetofection complexes contributes to efficient internalization of magnetofection complexes from the extracellular matrix into the cells. Therefore, we can expect the improved therapeutic effect as a consequence of effective accumulation of magnetofection complexes in the cells after magnetofection.

\section{The type of cells present in B16F10 tumor} model \& their uptake of magnetofection complexes

Melanoma tumors in this study were induced by injecting homogenous B16F10 cell suspension subcutaneously. Normally by increasing tumor cell mass also the extracellular matrix with recruited fibroblasts, endothelial cells, smooth muscle cells, and immune cells is formed, and further as the tumor mass exceeds $2 \mathrm{~mm}$ in diameter the vascular networks begin to establish [30]. Due to the heterogeneity in tumors, we examined various cell types for their ability to internalize magnetofection complexes.

On ultrathin sections of B16F10 tumors we could differentiate melanoma cells from immune, endothelial, muscle and nerve cells (Figure 4), which are present also in melanoma in man [31]. The ultrastructure of melanoma cells varied significantly with regard to the amount of melanosomes present in the cytoplasm (Figures 4A \& B), which is in agreement with the previous studies [31,32]. Melanosomes were of different maturation stages, and were present individually or clustered. In melanoma cells, magnetofection complexes were observed (Figure 4A), although also sections containing melanoma cells without internalized magnetofection complexes were noticed. (Figure 4B). Magnetofection complexes were seen in membrane compartments either individually distributed or in aggregates in the vicinity of the nucleus (Figures 3A \& 4A). Due to the technical limitations of TEM studies on whole tumors, the early steps of magnetofection complexes internalization is almost impossible to detect. However, previous studies have shown that magnetic nanoparticles are generally internalized by phagocytosis or micropinocytosis, and delivered to endosomes and lysosomes [3,33]. In addition to melanoma cells, magnetofection complexes were observed also in the immune cells, since some of them have the ability to phagocytose cell debris or magnetofection complexes directly (Figure 4C). In the endothelial, muscle and nerve cells magnetofection complexes were not noticed. It seems that melanoma cells are more susceptible to the uptake of magnetofection complexes than other cells present in the induced melanoma tumors. The reason is that tumor cells have high metabolic activity, therefore their uptake mechanisms are more pronounced in order to cover the needs for biosynthesis and their autonomous behavior [34]. This outcome is consistent with the results of previous in vitro studies demonstrating more effective uptake of magnetic nanoparticles into the tumor cells compared with normal $[3,8,35]$. In our previous studies we examined the transfection efficiency of magnetofection with pDNA encoding EGFP in different cell types including melanoma, mesothelial, endothelial, sarcoma and fibroblasts in vitro. The highest expression of EGFP was achieved in murine melanoma cells $[1,3-4,36]$.

TEM micrographs proved that magnetofection complexes are predominantly internalized by tumor melanoma cells, which are in fact our primary target for the therapy. The outcomes of this and our previous studies indicate that with magnetofection also selectivity could be achieved.

\section{The therapeutic effect of delivered plasmid DNA encoding shRNA against Mcam}

The data on the distribution and accumulation of magnetofection complexes within the melanoma tumors indicate that the exposure of tumors to an external magnetic field immediately after it. injection contributes to increased uptake of magnetofection complexes from the extracellular matrix into the cells. This could implicate that consequently the amount of cells, which contain therapeutic plasmid DNA is increased, and, therefore, it results in better therapeutic effectiveness. In order to determine that, we treated subcutaneous B16F10 tumors with three consecutive nanofections and magnetofections with therapeutic pDNA ${ }^{\text {anti-MCAM }}$, and monitored their growth.

The results demonstrated that only after magnetofection of B16F10 tumors with pDNA ${ }^{\text {anti-MCAM }}$ the significant therapeutic effect occurred (Figure 5A). The antitumor effect of magnetofection was noticed after 2nd therapy, leading to a statistically significant reduction of tumor volume compared with nanofection for 5 days, and resulting in $3.3 \pm 0.9$ days tumor growth delay compared with control group. In contrast to magnetofection, nanofection of B16F10 tumors with 


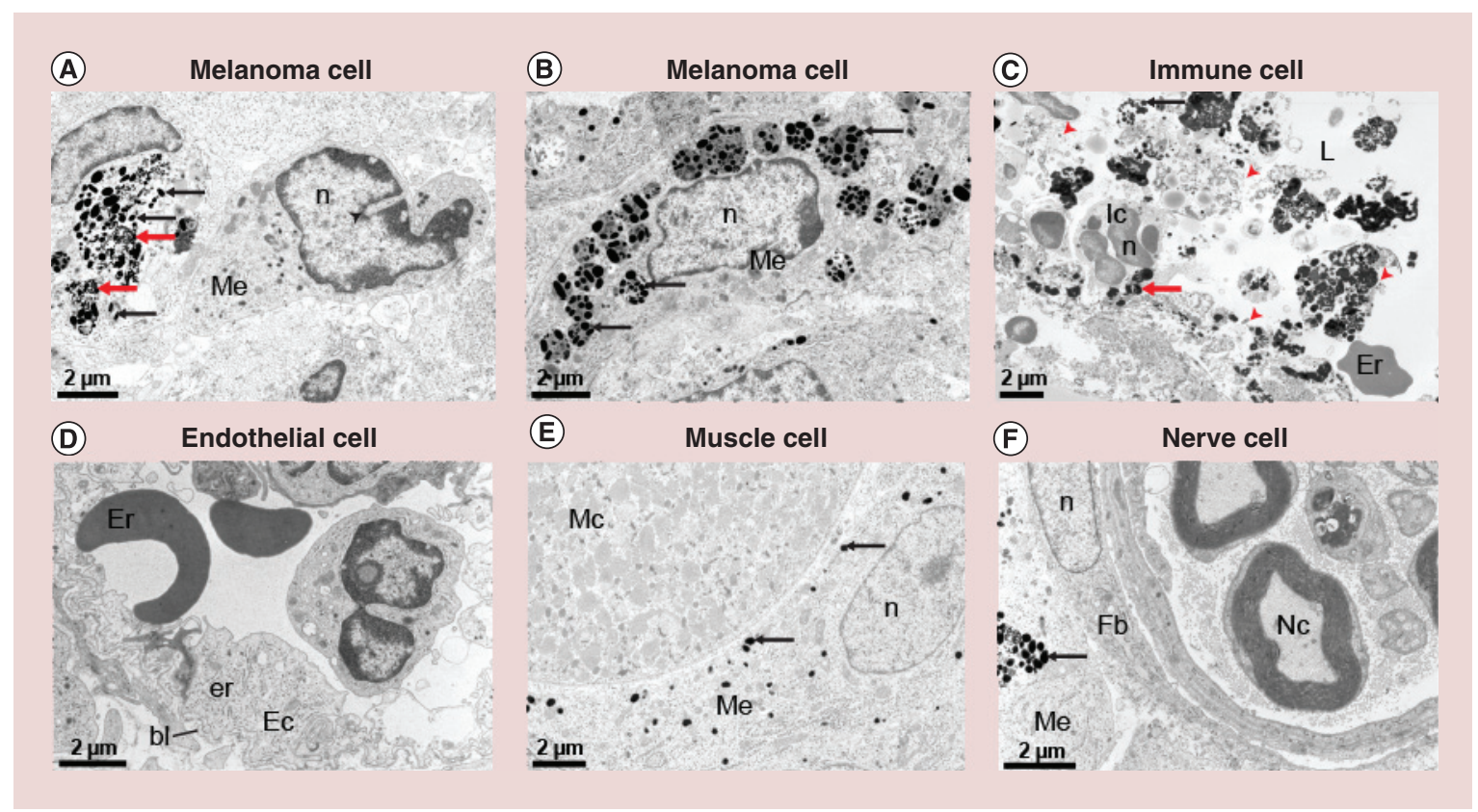

Figure 4. Transmission electron microscopy micrographs of ultrathin B16F10 tumor sections presenting different type of cells and their uptake of magnetofection complexes $24 \mathrm{~h}$ after 3 rd magnetofection. (A) Me contained melanosomes (black arrows) and internalized magnetofection complexes (red arrows). Among Me also invaginations of nucleus (black arrowhead) characteristic for tumors were seen. (B) Me varied in the amount of melanosomes, and some were filled with clusters of melanosomes (black arrows). (C) Ic present at the site of necrosis (red arrow-heads - cell debris) were phagocytozing cell debris. The debris contained melanosomes (black arrow) and magnetofection complexes (red arrow). (D-F) In the Ec, Mc, Nc, as well as in the Er and Fb magnetofection complexes were not noticed. Black arrows: melanosomes.

bl: Basal lamina; Ec: Endothelial cells; er: Endoplasmic reticulum; Er: Erythrocytes; Fb: Fibroblasts; Ic: Immune cells; L: Lumen, Mc: Muscle cells; Me: Melanoma cells; n: Nucleus; Nc: Nerve cells.

pDNA $^{\text {anti-MCAM }}$ had no antitumor effect. These results were consistent with the data presented in our previous study, where we also demonstrated a significant antitumor effect only when mammary adenocarcinoma (TS/A) tumors were exposed to an external magnetic field after it. injection of magnetofection complexes with bound $\mathrm{pDNA}^{\mathrm{IL}-12}$, whereas in the absence of external magnetic field there was no antitumor effect. However, therapeutic effect of IL-12 in TS/A tumors was more pronounced than that of targeting MCAM in
B16F10 melanoma [3]. The difference in the obtained antitumor effectiveness can be attributed to the different therapeutic plasmids used for the magnetofection of tumors, and the difference in the complexity and aggressiveness of treated tumor models.

In addition to monitoring tumor growth, a histological analysis of treated tumors was done after treatment of B16F10 tumors with pDNA ${ }^{\text {anti-MCAM }}$. Tumor sections from each experimental group were stained after 1 st and 3rd therapy with HE, rabbit polyclonal anti-

Figure 5. Therapeutic effect of magnetofection with plasmid DNA anti-MCAM on B16F10 tumors (see facing page). (A) The growth of B16F10 tumors and their growth delay after three consecutive intratumoral administrations of pDNA ${ }^{\text {anti-MCAM }}$ bound superparamagnetic iron oxide nanoparticles under the absence (NF) or presence (MF) of an external magnetic field. Arrows indicate the day of treatments. (B) The micrographs of B16F10 tumor sections stained with HE and the corresponding histological analysis. The presence of necrosis (orange arrows) and apoptosis (yellow arrows) in tumors after MF. Very few apoptotic cells were present after NF. (C) Transmission electron microscopy micrographs of ultrathin B16F10 tumor sections $24 \mathrm{~h}$ after 3rd magnetofection presenting cell necrosis and apoptosis. In the case of necrosis, the remains of cell (red arrow-heads) entering $L$ are frequently seen, and magnetofection complexes can be observed (red arrow) within the cell debris. In the case of apoptosis, cells with ultrastructural characteristic of apoptosis are seen: rounded, electron dense cells (red asterisk) with a bright belt of lumen (black arrow-heads) at the border with the neighboring cells. Arrows: melanosomes. (D) The micrographs of B16F10 tumor sections stained with antibodies against Ki-67 and the corresponding histological analysis. Ki-67-positive cells were counted in five acquired images of viable tumor tissue per each mouse in every experimental group and at both time points. Afterwards the percentage of proliferating cells normalized to the CTRL was determined. All quantitative data are expressed as arithmetic mean and standard error.

$* p<0.05$.

an: Nucleus of apoptotic cell; CTRL: Control group; HE: Hematoxylin and eosin; L: Lumen; MF: Magnetofection; n: Nucleus; NF: Nanofection; NSD: No statistically significant differences. 
(A)

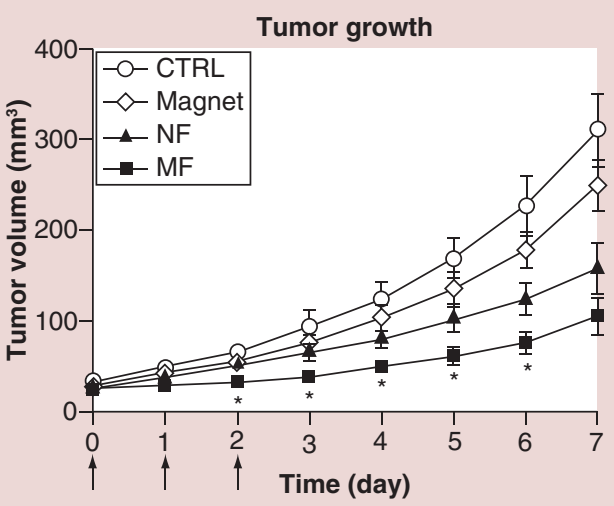

(B)

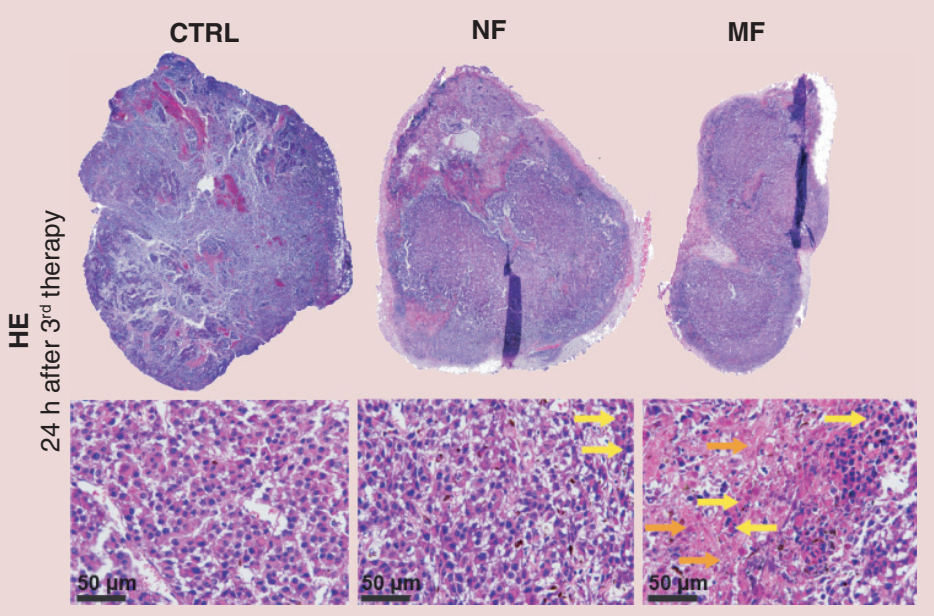

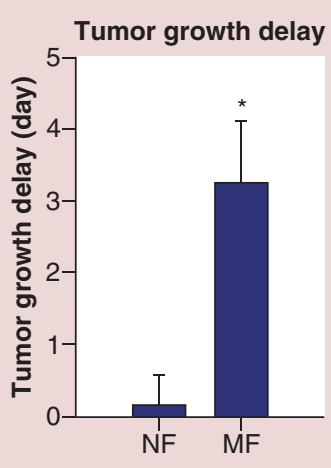

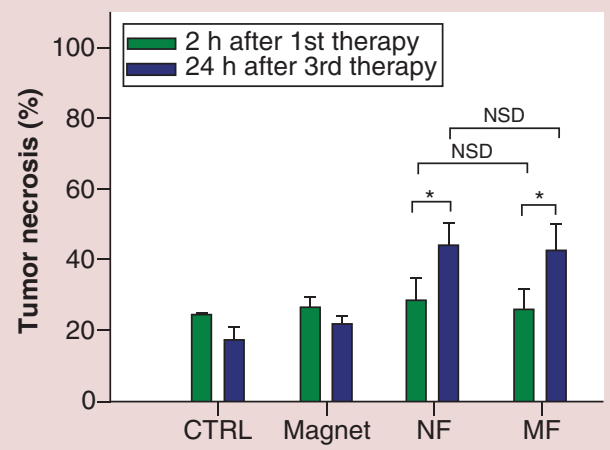

Cell apoptosis

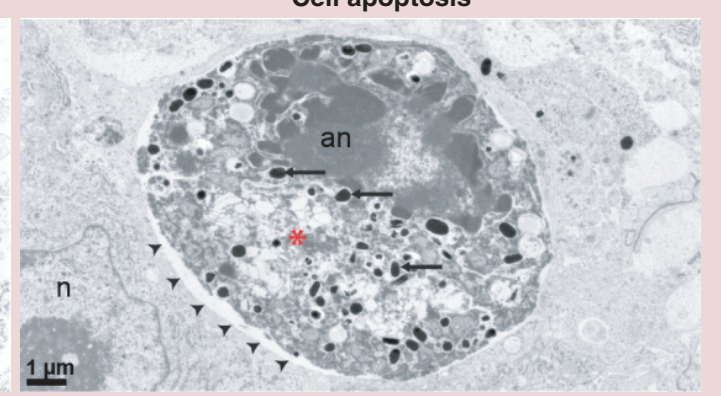

(D)
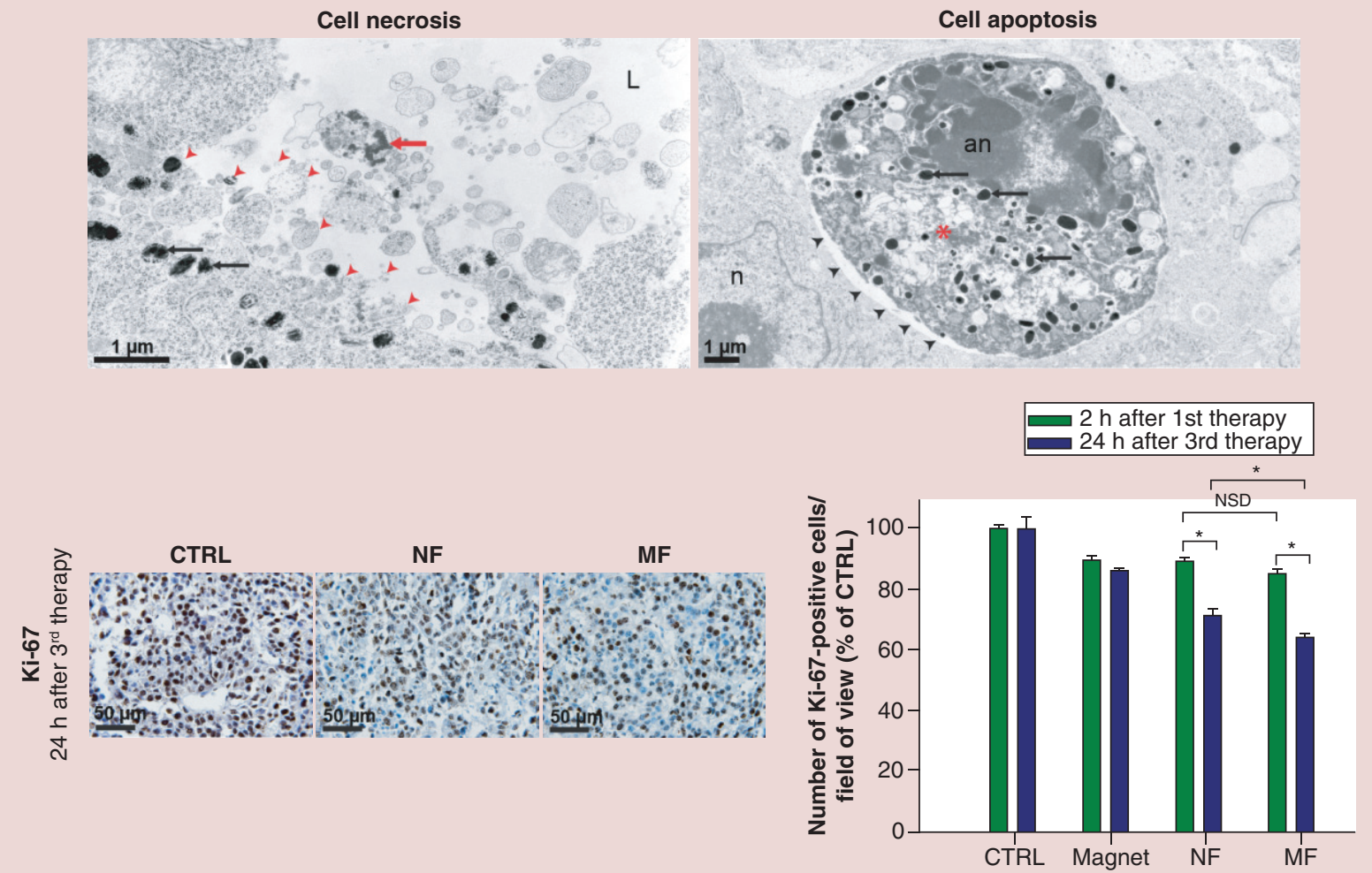
bodies against murine CD31-positive blood vessels and rabbit monoclonal antibodies against Ki-67-positive cells (a cellular marker for proliferation). Afterwards, the extent of tumor necrosis, the vascular density and the proliferation activity of tumor cells were analyzed. The sections were incubated with rabbit polyclonal antibodies against murine CD31 (ab28364, Abcam, MA, USA) at a dilution 1:1000 or rabbit monoclonal antibodies against Ki-67 (clone SP6, Thermo Fisher Scientific, MA, USA) at dilution 1:1200.

Three consecutive treatments with $\mathrm{pDNA}^{\text {anti-MCAM }}$ resulted in a statistically significantly increased tumor necrosis compared with the control group (Figure 5B), while $2 \mathrm{~h}$ after 1 st treatment no difference was noticed. Interestingly, we noticed extended areas with necrotic cells around magnetofection complexes $24 \mathrm{~h}$ after 3rd magnetofection, as we examined tumor sections stained with HE and Perl's Prussian blue at the same time (Supplementary Figure S1). However, the percentage of necrosis in tumors was estimated on $44 \%$ after nanofection and $43 \%$ after magnetofection, while in the control group on $25 \%$. Among nanofection and magnetofection there was no statistically significant difference noticed. We can assume that the obtained tumor necrosis $24 \mathrm{~h}$ after 3rd nanofection and magnetofection could be also due to the treatment merely, since the mechanical harms made by the needle and the injected volume of suspension of magnetofection complexes could not be excluded. Additionally, as the TEM micrographs of ultrathin tumor sections from the injection site $24 \mathrm{~h}$ after $3 \mathrm{rd}$ magnetofection were examined, the typical ultrastructural characteristics of the cells in necrosis or apoptosis were observed (Figure 5C).

In the remaining viable areas of tumors the CD31positive blood vessels were counted. The obtained results demonstrated no reduction in the number of CD31-positive blood vessels, implicating on vascular targeted effect after three consecutive therapies, neither after nanofection nor magnetofection (Supplementary Figure \$2). If we consider the fact that magnetofection complexes used for magnetofection were not effective in the transfection of endothelial cells in vitro neither with reporter or therapeutic plasmid DNA [1], the lack of vascular targeted effect after magnetofection of B16F10 tumors with pDNA anti-MCAM is comprehensible. Even from TEM micrographs of ultrathin B16F10 tumor sections presenting different type of cells $24 \mathrm{~h}$ after $3 \mathrm{rd}$ magnetofection, the magnetofection complexes in the endothelial cells were not noticed (Figure 4D). However, when using gene electrotransfer for the delivery of therapeutic plasmid DNA [1] or intraperitoneal injection for antibodies [22,24], the antiangiogenic effect after targeting MCAM in endo- thelial cells in vitro as well as in tumors in vivo has been proved $[1,22,24,37]$.

Finally, the Ki-67-positive cells were counted in the remaining viable tumor areas, and a statistically significant difference in the proliferation activity of tumor cells among nanofection and magnetofection was noticed (Figure 5D), indicating on antiproliferative mechanism of action of the therapy. The number of Ki-67-positive cells was reduced for $29 \%$ after 3 rd nanofection and for $36 \%$ after 3rd magnetofection in the comparison to the control group. The difference between antitumor effectiveness of nanofection and magnetofection would probably be greater if the histological analysis had been performed at later time points, since the reduction in tumor growth occurred only after the 3rd therapy. The late-onset of antitumor effect could be the consequence of long half-life of MCAM protein, which could be still present in the tumor cells, in spite of effective silencing of MCAM at the mRNA level $[1,38]$.

In this section we demonstrated that three consecutive magnetofections of B16F10 tumors with pDNA $^{\text {anti-MCAM }}$ resulted as an effective treatment. The histological analysis indicated that predominantly antiproliferative effect of MCAM silencing contributed to the antitumor effect. This outcome is consistent with the previous in vitro studies demonstrating antiproliferative effect after targeting MCAM with antibodies or RNA interference technology in tumor or endothelial cells [1,22,39-40].

\section{Conclusion}

The findings indicate that the it. injected magnetofection complexes retained locally at the injection site and did not distribute throughout the whole tumor, neither in a day after the last treatment. Further, we demonstrated that the exposure of tumors to an external magnetic field immediately after it. injection significantly contributes to the increased uptake of magnetofection complexes from the extracellular matrix into the cells. Among all the cell types present in the induced melanoma tumors, magnetofection complexes were internalized only by melanoma and immune cells. This outcome indicates the selectivity of magnetofection. Finally, three consecutive magnetofections of murine melanoma with $\mathrm{pDNA}^{\text {anti-MCAM }}$ resulted in marked antitumor effect.

\section{Future perspective}

In this study we aimed to explore, gain and update the knowledge about magnetofection, a developing nonviral gene delivery method. Its stage of development is still preclinical, and only few studies demonstrate its value in in vivo tumor models. As nonviral, non- 
invasive and painless gene delivery system, it certainly holds perspectives.

Our study demonstrates the importance of magnetic field for the antitumor effectiveness of in vivo magnetofection with therapeutic plasmid DNA. Therefore, further studies on the optimization of magnetic field distribution for in vivo magnetofection of tumors, could contribute to the improvement of magnetofection.

\section{Acknowledgements}

The authors thank Mira Lavric and Simona Kranjc from The Institute of Oncology Ljubljana, and Linda Strus from The Institute of Cell Biology in Ljubljana for all the work they contributed in this research.

\section{Financial \& competing interests disclosure}

Research was conducted in the scope of LEA EBAM (FrenchSlovenian European Associated Laboratory: Pulsed Electric Fields Applications in Biology and Medicine) and COST Action TD1104. The authors acknowledge the financial support from the state budget by the Slovenian Research Agency (program no. P3-0003, project no. J3-4211, J3-4259). The authors have no other relevant affiliations or financial involvement with any organization or entity with a financial interest in or fi- nancial conflict with the subject matter or materials discussed in the manuscript apart from those disclosed.

No writing assistance was utilized in the production of this manuscript.

\section{Ethical conduct of research}

All procedures were performed in the compliance with the guidelines for animal experiments of the EU directive (2010/63/EU) and the permission from the Veterinary Administration of the Ministry of Agriculture, Forestry and Food of the Republic of Slovenia (permission no. 34401-4/2012/2) and according to the standards stated in the 8th edition of the Guide for the Care and Use of Laboratory Animals. The authors state that they have obtained appropriate institutional review board approval or have followed the principles outlined in the Declaration of Helsinki for all human or animal experimental investigations. In addition, for investigations involving human subjects, informed consent has been obtained from the participants involved.

\section{Open access}

This work is licensed under the Attribution-Non Commercial-No Derivatives 4.0 Unported License. To view a copy of this license, visit http://creativecommons.org/licenses/by-nc-nd/4.0/

\section{Executive summary}

- Magnetofection as a nonviral gene delivery method is effective in the treatment of melanoma tumors in mice in vivo with plasmid DNA encoding short hairpin RNA against Mcam (pDNA anti-MCAM).

- The increased cellular uptake of magnetofection complexes after intratumoral injection was obtained by the exposure of tumors in vivo to an external magnetic field. The magnetic field significantly contributes to the uptake of magnetofection complexes from the extracellular matrix into the cells.

- The predominant cell types present in melanoma tumors, which accumulate magnetofection complexes, were melanoma and immune cells. This outcome indicates that with magnetofection also selectivity could be achieved.

- The antitumor effectiveness of three consecutive magnetofections of B16F10 tumors in mice in vivo with PDNA anti-MCAM was demonstrated by significant reduction of tumor volume and tumor growth delay. For greater effectiveness, consecutive magnetofections are needed, by providing more uniform distribution of magnetofection complexes and consequent transfection throughout the whole tumors.

\section{References}

Papers of special note have been highlighted as: • of interest; •• of considerable interest

1 Prosen L, Markelc B, Dolinsek T, Music B, Cemazar M, Sersa G. Mcam silencing with RNA interference using magnetofection has antitumor effect in murine melanoma. Mol. Ther. Nucleic Acids 3, e205 (2014).

2 Plank C, Zelphati O, Mykhaylyk O. Magnetically enhanced nucleic acid delivery. Ten years of magnetofection-progress and prospects. Adv. Drug Deliv. Rev. 63(14-15), 1300-1331 (2011).

- In this review, the progress and achievements in 10 years of magnetofection are summarized.

3 Prijic S, Prosen L, Cemazar M et al. Surface modified magnetic nanoparticles for immuno-gene therapy of murine mammary adenocarcinoma. Biomaterials 33(17), 4379-4391 (2012).

-• Our previous study demonstrating the effective uptake and uptake mechanism of SPIONs-PAA-PEI-pDNA (magnetofection complexes) in the different cell types in vitro after magnetofection by TEM and ICP-MS quantification method.

4 Prosen L, Prijic S, Music B, Lavrencak J, Cemazar M, Sersa G. Magnetofection: a reproducible method for gene delivery to melanoma cells. Biomed. Res. Int. 2013, 209452 (2013).

5 Ma Y, Zhang Z, Wang X, Xia W, Gu H. Insights into the mechanism of magnetofection using MNPs-PEI/pDNA/ free PEI magnetofectins. Int. J. Pharm. 419(1-2), 247-254 (2011). 
6 Zhu XM, Wang YX, Leung KC et al. Enhanced cellular uptake of aminosilane-coated superparamagnetic iron oxide nanoparticles in mammalian cell lines. Int. J. Nanomedicine 7, 953-964 (2012).

7 Kou L, Sun J, Zhai Y, He Z. The endocytosis and intracellular fate of nanomedicines: Implication for rational design. AJPS 8(1), 1-10 (2013).

8 Prijic S, Scancar J, Romih R et al. Increased cellular uptake of biocompatible superparamagnetic iron oxide nanoparticles into malignant cells by an external magnetic field. J. Membr. Biol. 236(1), 167-179 (2010).

9 Tran N, Webster TJ. Understanding magnetic nanoparticle osteoblast receptor-mediated endocytosis using experiments and modeling. Nanotechnology 24(18), 185102 (2013).

10 Huth S, Lausier J, Gersting SW et al. Insights into the mechanism of magnetofection using PEI-based magnetofectins for gene transfer. J. Gene Med. 6(8), 923-936 (2004).

11 Harush-Frenkel O, Rozentur E, Benita S, Altschuler Y. Surface charge of nanoparticles determines their endocytic and transcytotic pathway in polarized MDCK cells. Biomacromolecules 9(2), 435-443 (2008).

12 Singh N, Jenkins GJ, Asadi R, Doak SH. Potential toxicity of superparamagnetic iron oxide nanoparticles (SPION). Nano Rev. PMC3215220 (2010).

13 Petters C, Irrsack E, Koch M, Dringen R. Uptake and metabolism of iron oxide nanoparticles in brain cells. Neurochem. Res. 39(9), 1648-1660 (2014).

14 Prijic S, Sersa G. Magnetic nanoparticles as targeted delivery systems in oncology. Radiol. Oncol. 45(1), 1-16 (2011).

15 Delyagina E, Schade A, Scharfenberg D et al. Improved transfection in human mesenchymal stem cells: effective intracellular release of pDNA by magnetic polyplexes. Nanomedicine (Lond.) 9(7), 999-1017 (2014).

16 Thomas M, Klibanov AM. Non-viral gene therapy: polycation-mediated DNA delivery. Appl. Microbiol. Biotechnol. 62(1), 27-34 (2003).

17 Arsianti M, Lim M, Marquis CP, Amal R. Assembly of polyethylenimine-based magnetic iron oxide vectors: insights into gene delivery. Langmuir 26(10), 7314-7326 (2010).

18 Arsianti M, Lim M, Marquis CP, Amal R. Polyethylenimine based magnetic iron-oxide vector: the effect of vector component assembly on cellular entry mechanism, intracellular localization, and cellular viability. Biomacromolecules 11(9), 2521-2531 (2010).

19 Giustini AJ, Ivkov R, Hoopes PJ. An in vivo transmission electron microscopy study of injected dextran-coated ironoxide nanoparticle location in murine breast adenocarcinoma tumors versus time. Proc. SPIE Int. Soc. Opt. Eng. 7181, 71810M (2009).

-. One of the few studies examining the exact location of magnetic nanoparticles within the tumor after intratumoral injection.

20 Giustini AJ, Ivkov R, Hoopes PJ. Magnetic nanoparticle biodistribution following intratumoral administration. Nanotechnology 22(34), 345101 (2011).
21 Chen EY, Hodge S, Tai K et al. Oxygen microenvironment affects the uptake of nanoparticles in head and neck tumor cells. Proc. SPIE Int. Soc. Opt. Eng. 8584, 85840F (2013).

22 Yan X, Lin Y, Yang D et al. A novel anti-CD146 monoclonal antibody, AA98, inhibits angiogenesis and tumor growth. Blood 102(1), 184-191 (2003).

23 McGary EC, Heimberger A, Mills L et al. A fully human antimelanoma cellular adhesion molecule/MUC18 antibody inhibits spontaneous pulmonary metastasis of osteosarcoma cells in vivo. Clin. Cancer Res. 9(17), 6560-6566 (2003).

24 Mills L, Tellez C, Huang S et al. Fully human antibodies to MCAM/MUC18 inhibit tumor growth and metastasis of human melanoma. Cancer Res. 62(17), 5106-5114 (2002).

25 Herlyn M, Fukunaga-Kalabis M. What is a good model for melanoma? J. Invest. Dermatol. 130(4), 911-912 (2010).

26 Durymanov MO, Rosenkranz AA, Sobolev AS. Current approaches for improving intratumoral accumulation and distribution of nanomedicines. Theranostics 5(9), 1007-1020 (2015).

27 Mesojednik S, Pavlin D, Sersa G et al. The effect of the histological properties of tumors on transfection efficiency of electrically assisted gene delivery to solid tumors in mice. Gene Ther. 14(17), 1261-1269 (2007).

28 Johannsen M, Jordan A, Scholz R et al. Evaluation of magnetic fluid hyperthermia in a standard rat model of prostate cancer. J. Endourol. 18(5), 495-500 (2004).

29 Miao L, Zhang K, Qiao C et al. Antitumor effect of human TRAIL on adenoid cystic carcinoma using magnetic nanoparticle-mediated gene expression. Nanomedicine 9(1), 141-150 (2013).

30 Narang AS, Varia S. Role of tumor vascular architecture in drug delivery. Adv. Drug Deliv. Rev. 63(8), 640-658 (2011)

31 Curran RC, McCann BG. The ultrastructure of benign pigmented naevi and melanocarcinomas in man. J. Pathol. 119(3), 135-146 (1976).

- The examination of the structure of melanoma tumors in man by transmission electron microscopy.

32 Konrad K, Wolff K, Hönigsmann H. The giant melanosome: a model of deranged melanosome-morphogenesis. J. Ultrastruct. Res. 48(1), 102-123 (1974).

33 Kralj S, Rojnik M, Romih R, Jagodic M, Kos J, Makovec D. Effect of surface charge on the cellular uptake of fluorescent magnetic nanoparticles. J. Nanopart. Res. 14(1151), 1-14 (2012).

34 Mathew R, White E. Autophagy, stress, and cancer metabolism: what doesn't kill you makes you stronger. Cold. Spring Harb. Symp. Quant. Biol. 76, 389-396 (2011).

35 Ma YJ, Gu HC. Study on the endocytosis and the internalization mechanism of aminosilane-coated $\mathrm{Fe}_{3} \mathrm{O}_{4}$ nanoparticles in vitro. J. Mater. Sci. Mater. Med. 18(11), 2145-2149 (2007).

36 Prosen L, Cemazar M, Sersa G. Magnetofection: an effective, selective and feasible non-viral gene delivery method. IFMBE Proceedings. Springer, 335-338 (2015). 
Zeng Q, Wu Z, Duan $\mathrm{H}$ et al. Impaired tumor angiogenesis and VEGF-induced pathway in endothelial CD146 knockout mice. Protein Cell 5(6), 445-456 (2014).

38 Pai SI, Lin YY, Macaes B, Meneshian A, Hung CF, Wu TC. Prospects of RNA interference therapy for cancer. Gene Ther. 13(6), 464-477 (2006).

39 Kang Y, Wang F, Feng J, Yang D, Yang X, Yan X. Knockdown of CD146 reduces the migration and proliferation of human endothelial cells. Cell Res. 16(3), 313-318 (2006).

40 Chen W, Zhang HL, Jiang YG, Li JH, Liu BL, Sun MY. Inhibition of CD146 gene expression via RNA interference reduces in vitro perineural invasion on ACC-M cell. J. Oral Pathol. Med. 38(2), 198-205 (2009). 